\title{
Patterns and diagnostic criteria of necrotizing fasciitis
}

\author{
Muhammed Ihsan Muhammad ${ }^{1 *}$, Mezzher Mohammad Alsaeed ${ }^{2}$, Ali Abdulghalib Alhayek ${ }^{2}$, \\ Layla Hassan Alnosair², Aqeel Salman Al Alkhazal², Amar Mustafa Alhaji², \\ Mohammed Abdullah Mersil ${ }^{3}$, Amina Mohamed Hamid ${ }^{4}$, Abdulaziz Hussain Alfoudari ${ }^{5}$, \\ Huda Ayedh AlGossadi ${ }^{6}$, Aisha Jaber Asiri ${ }^{7}$, Fatma Abdulla Al-Fodari ${ }^{5}$
}

\author{
${ }^{1}$ Department of General Surgery, Khulais General Hospital, Khulais, Saudi Arabia \\ ${ }^{2}$ Department of Pediatric Surgery, Maternity and Children Hospital, Dammam, Saudi Arabia \\ ${ }^{3}$ Department of General Surgery, Ministry of Health, Kuwait, Kuwait \\ ${ }^{4}$ Department of General Surgery, Security Forces Hospital, Mecca, Saudi Arabia \\ ${ }^{5}$ Department of General Surgery, Adan Hospital, Hadiya, Kuwait \\ ${ }^{6}$ Department of General Surgery, Armed Forces Hospital Southern Region, Abha, Saudi Arabia \\ ${ }^{7}$ Department of General Surgery, King Fahad Armed Forces Hospital, Abha, Saudi Arabia
}

Received: 01 July 2021

Accepted: 15 July 2021

\section{*Correspondence:}

Dr. Muhammed Ihsan Muhammad,

E-mail: ihsan863@yahoo.com

Copyright: () the author(s), publisher and licensee Medip Academy. This is an open-access article distributed under the terms of the Creative Commons Attribution Non-Commercial License, which permits unrestricted non-commercial use, distribution, and reproduction in any medium, provided the original work is properly cited.

\begin{abstract}
Although the condition is not common, if the diagnosis of necrotizing fasciitis was established late, many lifethreatening complications might develop as sepsis and septic shock, which might lead to multiorgan damage. In the present literature review, we aim to discuss the classification and clinical patterns of necrotizing fasciitis, in addition to the diagnostic criteria and modalities that were reported among studies in the literature to evaluate such cases. Two main types of necrotizing fascitis were reported in the literature, including the poly and monomicrobial types, however, the diagnostic criteria for each are usually similar. Establishing an early diagnosis is essential to achieve better management and reduce the potential development of complications and death. The clinical patterns are the cornerstone for establishing the diagnosis, however, laboratory investigations might also be used as valid approaches to confirm the diagnosis. Many laboratory models have been proposed to establish the diagnosis of necrotizing fasciitis with variable sensitivities and specificities, and the laboratory risk indicator for necrotizing fasciitis (LRINEC) remains the commonest most efficacious modality. A tissue biopsy can also be used within the clinical settings for indicating the infection, however, it should not hinder the intended surgical interventions. Studies also show that magnetic resonance imaging can adequately detect liquefactive necrosis and is reported with a higher sensitivity than computed tomography. Although the condition is not very common, it might lead to severe consequences, and therefore, early extensive treatment and interventional approaches are encouraged.
\end{abstract}

Keywords: Clinical, Infection, Necrotizing fasciitis, Diagnosis

\section{INTRODUCTION}

Over the years, necrotizing fascitis has been given many names as phagedenic gangrenosum, phagedena, nonclostridial gas gangrene, and synergistic, progressive bacterial gangrene. ${ }^{1}$ It was first reported in the United
States by Joseph Jones with an approximate death rate of $50 \% .^{2}$ Later on, in 1883 , the first pathological remarks of the disease affecting external genitalia and perineum were published by Fournier. ${ }^{3}$ In 1924, hemolytic streptococci were identified as causes of the condition. ${ }^{4}$ The term necrotizing fascitis was first reported by Wilson, which identified the disease as severe infection and 
inflammation occurring mainly to the deep fascia surrounding the muscular tissues. ${ }^{5}$ Many etiologies have been proposed for the condition, including surgical incisions, traumas, insect bites, contusions, abrasions, and others. ${ }^{5-7}$ Although the condition is not common, if the diagnosis was established late, many life-threatening complications might develop as sepsis and septic shock, which might lead to multiorgan damage.$^{8-10}$ In the present literature review, we aim to discuss the classification and clinical patterns of necrotizing fasciitis, in addition to the diagnostic criteria and modalities that were reported among studies in literature to evaluate such cases.

We performed an extensive literature search of the Medline, Cochrane, and Embase databases on $18^{\text {th }}$ June 2021 using the medical subject headings (MeSH) or a combination of all possible related terms. This was followed by the manual search for papers in Google scholar and the reference lists of the initially included papers. ${ }^{11,12}$ Papers discussing the classification and clinical patterns of necrotizing fasciitis were screened for relevant information. We did not pose any limits on date, language, age of participants, or publication type.

\section{DISCUSSION}

\section{Classification and clinical patterns}

Necrotizing fasciitis is generally known as a serious infection that usually affects the soft tissue, which can subsequently induce serious damage to the fascia of the underlying muscles and the subcutaneous fat. Among the different studies in the literature, many classification modalities were proposed, however, among the various modalities, two classifications of necrotizing fasciitis are generally used within the clinical settings. ${ }^{13}$ Based on the detected bacteria within the infected region, the two main types and classifications of necrotizing fascitis have been proposed. ${ }^{13,14}$ In type I necrotizing fasciitis, the disease is usually called polymicrobial due to the variously detected microbiological organisms during diagnosis. Clinical and laboratory investigations indicated that both anaerobic and aerobic bacterial pathogens are commonly identified in this type of necrotizing fasciitis. Similar to the clinical observations with gas gangrene, this type of infection has also been noticed to cause gaseous-like infiltration and pathology of the infected tissue. ${ }^{15,16}$ Besides, type I accounts for the most common forms of necrotizing fascitis and usually affects patients that are old and with chronic disorders. On the other hand, type II necrotizing fasciitis, also known as monomicrobial, is when the infection occurs with a single organism which is usually methicillin-resistant Staphylococcus aureus or Grampositive organisms such as group A Streptococcus. This type has been reported to occur with a toxic shock syndrome that is attributable to the endotoxins released from the causative bacteria. ${ }^{15-17}$ No age groups or risk factors have been linked with the development of type II, unlike type I. ${ }^{16,17}$ Klebsiella, Pseudomonas, Clostridium, Vibrio vulnificus, and Aeromonas are rarely observed to cause necrotizing fascitis, however, when the infection by these organisms occurs, the case is usually severe and requires dedicated medical approaches to manage. Besides, previous studies have suggested that infection with these types of bacteria should be classified as a third type of necrotizing fascitis, however, the evidence is still not sufficient. ${ }^{16,18}$ Ludwig angina was also reported as another form of necrotizing fascitis that results from infection to the submandibular region. Lemierresyndrome was also reported to affect the internal jugular vein causing septic thrombophlebitis as a result of a predisposing oropharyngeal infection. Fournier gangrene was also reported as an infection to the gastrointestinal tract and urethra that might also discharge to the perineal area and is usually associated with gas infiltration. ${ }^{17,19}$

Within the clinical settings, patients with necrotizing fascitis might present with local superficial edema and erythema, ${ }^{20}$ which is usually similar to the manifestations of cellulitis, and therefore, the early differential diagnosis should be conducted to achieve better management. Organ damage, limb loss, and increased risk of mortality might be present in cases where the diagnosis and management of necrotizing fasciitis were delayed. ${ }^{16,21}$ Tissue necrosis that is preceded by the presence of hemodynamic instability associated with severe pain and tenderness might be the main manifestations that can be used clinically to differentiate necrotizing fasciitis from cellulitis. ${ }^{16}$ Elevated levels of aspartate aminotransferase and creatine kinase are also suggestive of the presence of a deep tissue injury. ${ }^{22}$ However, it should be noted that the differentiation between cellulitis and necrotizing fasciitis is still difficult within a clinical setting. ${ }^{16}$ It was previously suggested that using anti-inflammatory drugs and analgesics can help with establishing a proper diagnosis, as the pain that is associated with necrotizing fasciitis usually persists even after the administration of these modalities. However, conditions impairing a patient's sensations as diabetic neuropathy should be considered because these might lower the pain threshold of the condition. ${ }^{13,18,23}$ The site of the infection is also essential in the determination of the prognosis and severity of the case. For instance, infections occurring to the head and neck usually develop into mediastinitis due to polymicrobial affection. ${ }^{23-25}$ It should also be noted that the early clinical presentation might be mild or asymptomatic, as patients might only suffer from local inflammation or skin irritation. ${ }^{15,19}$ Pain, tenderness, edema, erythema, and fever are the commonest clinical patterns that are usually observed among patients with necrotizing fasciitis, irrespective of the severity or type of the infection. However, in type II, the clinical patterns might not develop within the early stages following the infection and might last for long periods when the prognosis becomes a poor and deep invasion of the infections occurs. ${ }^{14,15,19,26}$ Lactic acidosis and hemodynamic instability that is associated with septic shock or sepsis might also be a manifestation in patients with multiple chronic conditions and are infected with virulent organisms, which might lead to severe disease 
and multiorgan damage. ${ }^{17}$ Moreover, subcutaneous crepitus can be detected in some patients that are affected by gas-producing bacterial pathogens. Blisters, bullae, and other skin lesions are usually observed when the prognosis is poor and the infection is severe. However, it should be noted that the sensitivity of these events is not high, although they can be used to differentiate necrotizing fascitis from cellulitis. Therefore, the attending physicians should have a high index of suspicion to initiate the diagnosis of the condition, as many of these unspecific symptoms might even not develop in many cases. ${ }^{13-15,19,23,26}$ Although many classifications and etiologies for necrotizing fasciitis have been proposed, the overall diagnostic criteria are similar among them. ${ }^{16,21}$

\section{DIAGNOSIS}

The primary diagnosis of necrotizing fasciitis is clinical while other radiological and laboratory tests can be used to confirm the diagnosis. A comprehensive metabolic assessment, complete blood picture, and coagulation profiles are the main laboratory investigations that can be used in these events. Additionally, therapeutic laboratory assessments can also be done, as blood and tissue cultures, to identify the causative organisms and the best highly sensitive antibiotic therapy. When sepsis is clinically established, assessment of the arterial blood gases should be considered. ${ }^{5}$ Hypoproteinemia, hyponatremia, azotemia, hematuria, thrombocytopenia, high levels of erythrocyte sedimentation rates and creatine kinase, hyperbilirubinemia, hypoalbuminemia, anemia, and metabolic acidosis can be observed in patients with necrotizing fasciitis. Although metabolic findings might constitute an essential part of diagnosing the condition in the early stages, the reported findings are usually massively detectable when sepsis develops. For instance, hypocalcemia can only be detected in the early stages when fat necrosis is present. To establish a proper diagnosis of necrotizing fasciitis, regular follow-up by laboratory investigations should be adequately conducted to prevent any potential overlapping between the condition and other infections. A previous investigation by Wall et al developed a model for this purpose and showed that estimated levels of serum $\mathrm{Na}<135 \mathrm{mmol} / \mathrm{L}$, and white blood cells (WBCs) $>15,400$ cells $/ \mu \mathrm{L}$ might have an estimated high sensitivity of $90 \%$ for diagnosing necrotizing fasciitis. ${ }^{27}$ However, they also reported that the tool is not highly predictive of the infection, as the estimated positive predictive value and specificity were only $26 \%$, and $76 \%$, respectively. Another model was also developed for the diagnosis of necrotizing fasciitis caused by group A Streptococci. The estimated sensitivity and specificity for the tool when C-reactive protein (CRP) is $>18 \mathrm{mg} / \mathrm{dL}$ were $89 \%$ and $90 \%$, respectively, and $58 \%$, and $95 \%$, respectively, when the estimated creatine kinase levels $>600 \mathrm{U} / \mathrm{L}$ when used to differentiate necrotizing fascitis from cellulitis. ${ }^{28}$ The laboratory risk indicator for necrotizing fasciitis (LRINEC) was then proposed by Wong et al to differentiate between necrotizing fasciitis and other infections of the soft tissues, based on many laboratory parameters, including WBCs count, serum Na, hemoglobin, CRP, glucose, and creatinine levels (Figure 1). ${ }^{29}$ Assessment by the model was based on a scoring system that ranged between 0 and 13 , and a cutoff point of 6 for diagnosing necrotizing fasciitis was estimated to have negative and positive predictive values of $96 \%$, and $92 \%$, respectively. The authors also reported that the probability of necrotizing fasciitis was $>75 \%$ when the total score was 8 or more, $50-75 \%$ when the score was 6 7 , and less than $50 \%$ when the score was 5 or less.

\begin{tabular}{lll}
\hline Parameter & Range & Score $^{\mathbf{a}}$ \\
\hline $\mathrm{Hb}(\mathrm{g} / \mathrm{dl})$ & $>13.5$ & 0 \\
& $11-13.5$ & 1 \\
& $<11$ & 2 \\
& $<15$ & 0 \\
White cells $\left(10^{\prime} 9 / \mathrm{L}\right)$ & $<-25$ & 1 \\
& $>25$ & 2 \\
Sodium (mmol/L) & $<135$ & 2 \\
Creatinine (umol L) & $>141$ & 2 \\
Glucose & $>10$ & 1 \\
C-reactive protein & $>150$ & 4 \\
\hline
\end{tabular}

${ }^{a}$ Score $\leq 5=<50 \%$ risk (low); $6-7=$ intermediate risk; $\geq 8=>75 \%$ risk (high).

Figure 1: The laboratory risk indicator for necrotizing fasciitis (LRINEC) scoring system. ${ }^{29}$

Radiological assessments can be used for potential gas detection in the affected regions. However, the modality is not very sensitive in such cases as gas detection might require the development of tissue necrosis and the development of late-stage disease. Rather than plan radiographic modalities, computed tomography (CT) was proposed as a more efficacious modality. Fascial thickening in the affected regions, in addition to stranding with enhanced attenuation of the subcutaneous tissue, is the main characteristic that can be detected in up to $80 \%$ of the cases with necrotizing fasciitis. ${ }^{30} \mathrm{CT}$ images might present the presence of gas in between the tissues or the pathology might be present as tracking the corresponding fascia. ${ }^{30}$ Besides, these modalities can also be used for confirming the clinical patterns and boundaries of the infection, as previously noticed when detecting edema. However, previous estimates show that CT imaging is not very sensitive as many previous cases with necrotizing fasciitis were adequately diagnosed with clinical and laboratory investigations while the imaging results did not show any pathological markings. ${ }^{31}$ On the other hand, studies have demonstrated that the sensitivity with magnetic resonance imaging (MRI) modalities might constitute up to $100 \%$ when being used for the diagnosis of necrotizing fasciitis. ${ }^{31}$ Using T2-weighted imaging 
features, the pathology of necrotizing fasciitis might be present as increased focal intensities, referring to increased accumulation of abnormal fluids within the affected fascia, which indicates the presence of inflammatory edema and severe liquefactive tissue necrosis. Using T1-weighted imaging features, the pathology is usually present as an area of variable intensities within the thick affected fascia. ${ }^{32}$ It should be noted that MRI should not be used when the case is severe and the patient is hemodynamically unstable, and in such cases, surgical debridement should be approached instead. Tissue biopsy is another modality that can be used for confirming the diagnosis of necrotizing fasciitis. Finger test and frozen-section biopsies are previously validated modalities for such purposes. These modalities can significantly fasten the diagnosis process and decrease the period from diagnosis to onset of symptoms. However, clinical data indicate that the process is usually complex and requires a high level of experience, and therefore, it might not be always available, although it has been associated with reduced death rates due to early diagnosis. ${ }^{33}$ Although frozen-section biopsies are recommended to indicate necrotizing fasciitis in severe cases with hemodynamic instability, surgical interventions should not be delayed in such cases to enhance the prognosis.

\section{CONCLUSION}

In the present literature review, we have discussed the classification and clinical patterns of necrotizing fasciitis, in addition to the diagnostic criteria and modalities that were reported among studies in the literature to evaluate such cases. Two main types of necrotizing fascitis were reported in the literature, including the poly and monomicrobial types, however, the diagnostic criteria for each are usually similar. Establishing an early diagnosis is essential to achieve better management and reduce the potential development of complications and death. The clinical patterns are the cornerstone for establishing the diagnosis, however, laboratory investigations might also be used as valid approaches to confirm the diagnosis. Although the condition is not very common, it might lead to severe consequences, and therefore, early extensive treatment and interventional approaches are encouraged.

Funding: No funding sources Conflict of interest: None declared Ethical approval: Not required

\section{REFERENCES}

1. Wong $\mathrm{CH}$, Chang HC, Pasupathy S, Khin LW, Tan JL, Low CO. Necrotizing fasciitis: clinical presentation, microbiology, and determinants of mortality. J bone and joint surgery Am vol. 2003;85(8):1454-60.

2. Sadasivan J, Maroju NK, Balasubramaniam A. Necrotizing fasciitis. Indian $J$ Plast Surg. 2013;46(3):472-8.
3. Fournier JA. Jean-Alfred Fournier 1832-1914. Gangrène foudroyante de la verge (overwhelming gangrene). Sem Med 1883. Diseases of the colon and rectum. 1988;31(12):984-8.

4. MELeney FL. Hemolytic Streptococcus gangrene. Arch Surg. 1924;9(2):317-64.

5. Carter PS, Banwell PE. Necrotising fasciitis: a new management algorithm based on clinical classification. Int wound j. 2004;1(3):189-98.

6. Dufel S, Martino M. Simple cellulitis or a more serious infection? J family practice. 2006;55(5):396400.

7. Bisno AL, Stevens DL. Streptococcal infections of skin and soft tissues. N Engl J Med. 1996;334(4):240-5.

8. Cherneski CL, Embil JM. Necrotizing fasciitis. Saudi med J . 2001;22(7):565-8.

9. Meltzer DL, Kabongo M. Necrotizing fasciitis: a diagnostic challenge. Am Fam Physician. 1997;56(1):145-149.

10. Holmström B, Grimsley EW. Necrotizing fasciitis and toxic shock-like syndrome caused by group B Streptococcus. Southern med j. 2000;93(11):1096-8.

11. El-Qushayri AE, Khalaf KM, Dahy A. Fournier's gangrene mortality: A 17-year systematic review and meta-analysis. Int J Infect Dis. 2020;92:218-25.

12. Ghozy S, Tran L, Naveed S. Association of breastfeeding status with risk of autism spectrum disorder: A systematic review, dose-response analysis and meta-analysis. Asian $\mathbf{J}$ Psychiatr. 2020;48:101916.

13. Hakkarainen TW, Kopari NM, Pham TN, Evans HL. Necrotizing soft tissue infections: review and current concepts in treatment, systems of care, and outcomes. Curr Probl Surg. 2014;51(8):344-62.

14. Jabbour G, El-Menyar A, Peralta R. Pattern and predictors of mortality in necrotizing fasciitis patients in a single tertiary hospital. World $\mathrm{J}$ Emergency Surg. 2016;11.

15. Shiroff AM, Herlitz GN, Gracias VH. Necrotizing soft tissue infections. $\mathbf{J}$ intensive care med. 2014;29(3):138-44.

16. Stevens DL, Bryant AE. Necrotizing Soft-Tissue Infections. N Engl J Med. 2017;377(23):2253-65.

17. Misiakos EP, Bagias G, Patapis P, Sotiropoulos D, Kanavidis P, Machairas A. Current concepts in the management of necrotizing fasciitis. Frontiers in surg. 2014;1:36.

18. Henry S, Davis K, Morrison J, Scalea T. Can necrotizing soft tissue infection be reliably diagnosed in the emergency department? Trauma Surgery Acute Care Open. 2018;3:e00157.

19. Kiat HJ, En Natalie YH, Fatimah L. Necrotizing Fasciitis: How Reliable are the Cutaneous Signs? J emergencies, trauma, and shock. 2017;10(4):205-10.

20. Fontes RA, Jr, Ogilvie CM, Miclau T. Necrotizing soft-tissue infections. J Am Academy Orthop Surgeons. 2000;8(3):151-8.

21. Stevens DL, Bisno AL, Chambers HF. Practice guidelines for the diagnosis and management of skin 
and soft tissue infections: 2014 update by the Infectious Diseases Society of America. Clin Infect Dis. 2014;59(2): e10-52.

22. Montravers P, Snauwaert A, Welsch C. Current guidelines and recommendations for the management of skin and soft tissue infections. Current opinion in infectious diseases. 2016;29(2):131-8.

23. Wang JM, Lim HK. Necrotizing fasciitis: eight-year experience and literature review. Brazilian $\mathrm{j}$ infect diseases. 2014;18(2):137-43.

24. Zundel S, Lemaréchal A, Kaiser P, Szavay P. Diagnosis and Treatment of Pediatric Necrotizing Fasciitis: A Systematic Review of the Literature. Eur j pediatric surg. 2017;27(2):127-37.

25. Thieu H, Bach Dat B, Nam NH. Antibiotic resistance of Helicobacter pylori infection in a children's hospital in Vietnam: prevalence and associated factors. Minerva medica. 2020;111(5):498-501.

26. Bystritsky R, Chambers H. Cellulitis and Soft Tissue Infections. Ann Intern Med. 2018;168(3):Itc17-32.

27. Wall DB, Klein SR, Black S, de Virgilio C. A simple model to help distinguish necrotizing fasciitis from nonnecrotizing soft tissue infection. J Am Coll Surg. 2000;191(3):227-31.

28. Simonart T. Group a beta-haemolytic streptococcal necrotising fasciitis: early diagnosis and clinical features. Dermatology (Basel, Switzerland). 2004;208(1):5-9.
29. Wong CH, Khin LW, Heng KS, Tan KC, Low CO. The LRINEC (Laboratory Risk Indicator for Necrotizing Fasciitis) score: a tool for distinguishing necrotizing fasciitis from other soft tissue infections. Crit Care Med. 2004;32(7):1535-41.

30. Wysoki MG, Santora TA, Shah RM, Friedman AC. Necrotizing fasciitis: CT characteristics. Radiology. 1997;203(3):859-63.

31. Young $\mathrm{MH}$, Aronoff DM, Engleberg NC. Necrotizing fasciitis: pathogenesis and treatment. Expert review of anti-infective therapy. 2005;3(2):279-94.

32. Fugitt JB, Puckett ML, Quigley MM, Kerr SM. Necrotizing fasciitis. Radiographics : a review publication of the Radiological Society of North America, Inc. 2004;24(5):1472-6.

33. Anaya DA, Dellinger EP. Necrotizing soft-tissue infection: diagnosis and management. Clin Infect Dis. 2007;44(5):705-10.

Cite this article as: Muhammad MI, Alsaeed MM, Alhayek AA, Alnosair LH, Al Alkhazal AS, Alhaji AM et al. Patterns and diagnostic criteria of necrotizing fasciitis. Int J Community Med Public Health 2021;8:4062-6. 